Supporting Information

\title{
Branched poly-L-lysine derived nitrogen-containing porous carbon flake as the metal-free electrocatalyst towards efficient oxygen reduction reaction
}

Jiakang Min, ${ }^{a *}$ Xiaodong Xu, J. Justin Koh, ${ }^{a}$ Jiang Gong, ${ }^{c *}$ Xuecheng Chen, ${ }^{b}$ Jalal Azadmanjiri, ${ }^{d}$ Feifei Zhang, ${ }^{a}$ Xin Wen, ${ }^{b}$ and Chaobin He ${ }^{a *}$

a Department of Materials Science \& Engineering, National University of Singapore, 9 Engineering Drive 1, Singapore 117576, Singapore.

b Nanomaterials Physicochemistry Department, Faculty of Chemical Technology and Engineering, West Pomeranian University of Technology in Szczecin, Piastów Avenue 42, Szczecin 71065, Poland.

${ }^{c}$ Key Laboratory of Material Chemistry for Energy Conversion and Storage, Ministry of Education, Hubei Key Laboratory of Material Chemistry and Service Failure, Hubei Engineering Research Center for Biomaterials and Medical Protective Materials, School of Chemistry and Chemical Engineering, Huazhong University of Science and Technology, Wuhan 430074, China.

d Department of Inorganic Chemistry, University of Chemistry and Technology Prague, Technická 5, 16628 Prague 6, Czech Republic.

*Corresponding to J. Min (msemj@ nus.edu.sg, mjkxxd@gmail.com), J. Gong (gongjiang@ hust.edu.cn), C. He (․ㅡsehc@ nus.edu.sg) 

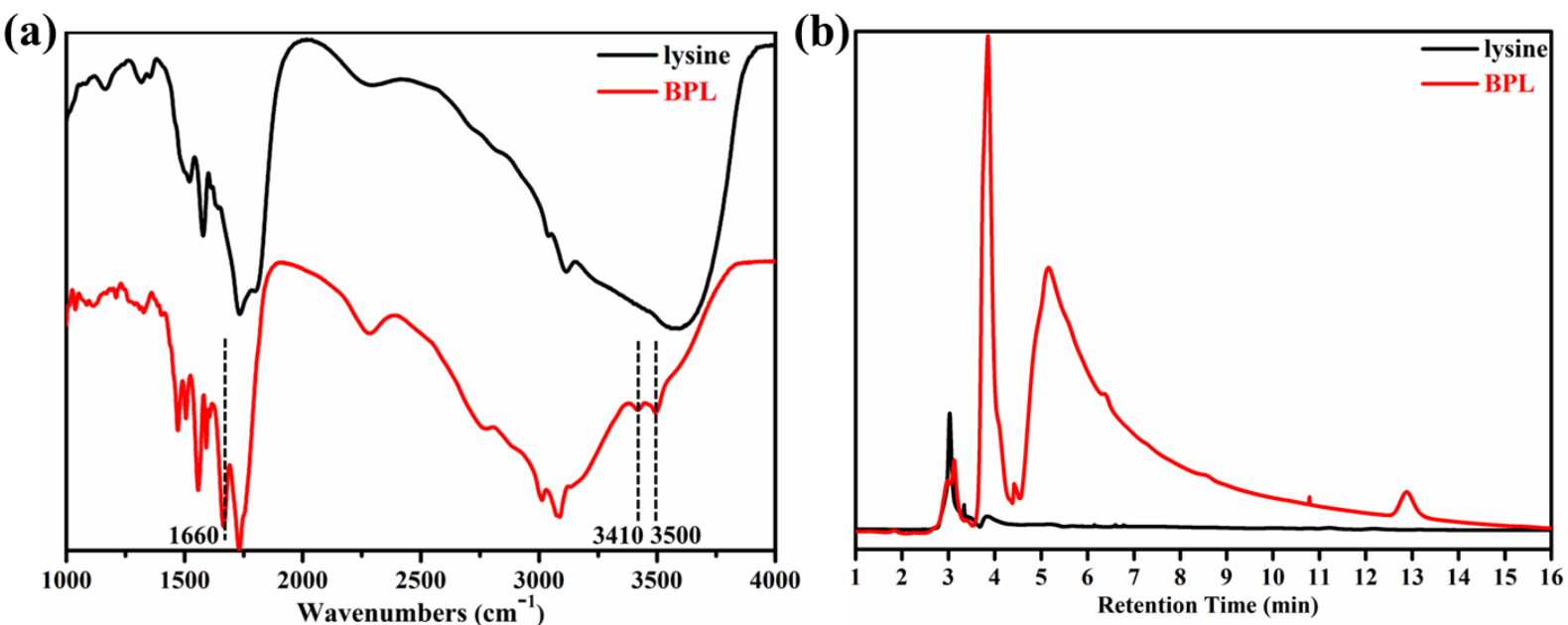

Figure S1. (a) FTIR spectrum and (b) high-performance liquid chromatogram of lysine and BPL.

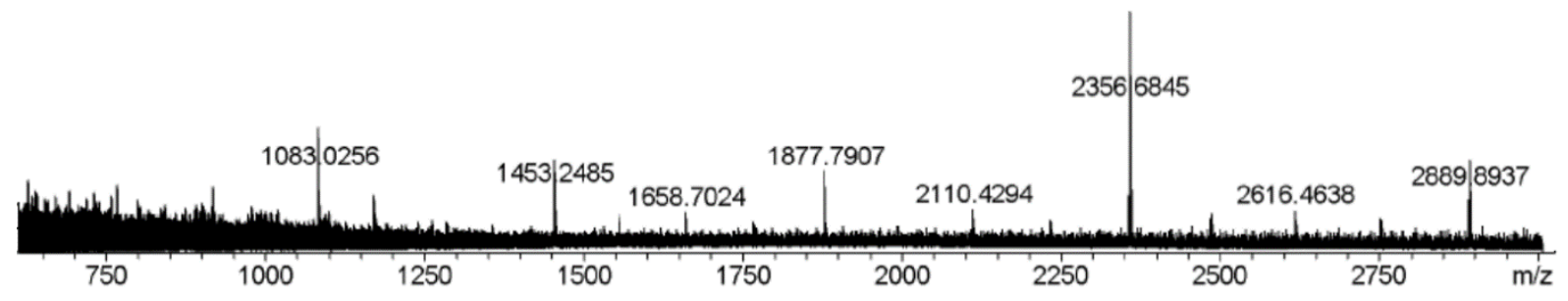

Figure S2. MALDI-TOF spectrum of BPL. 

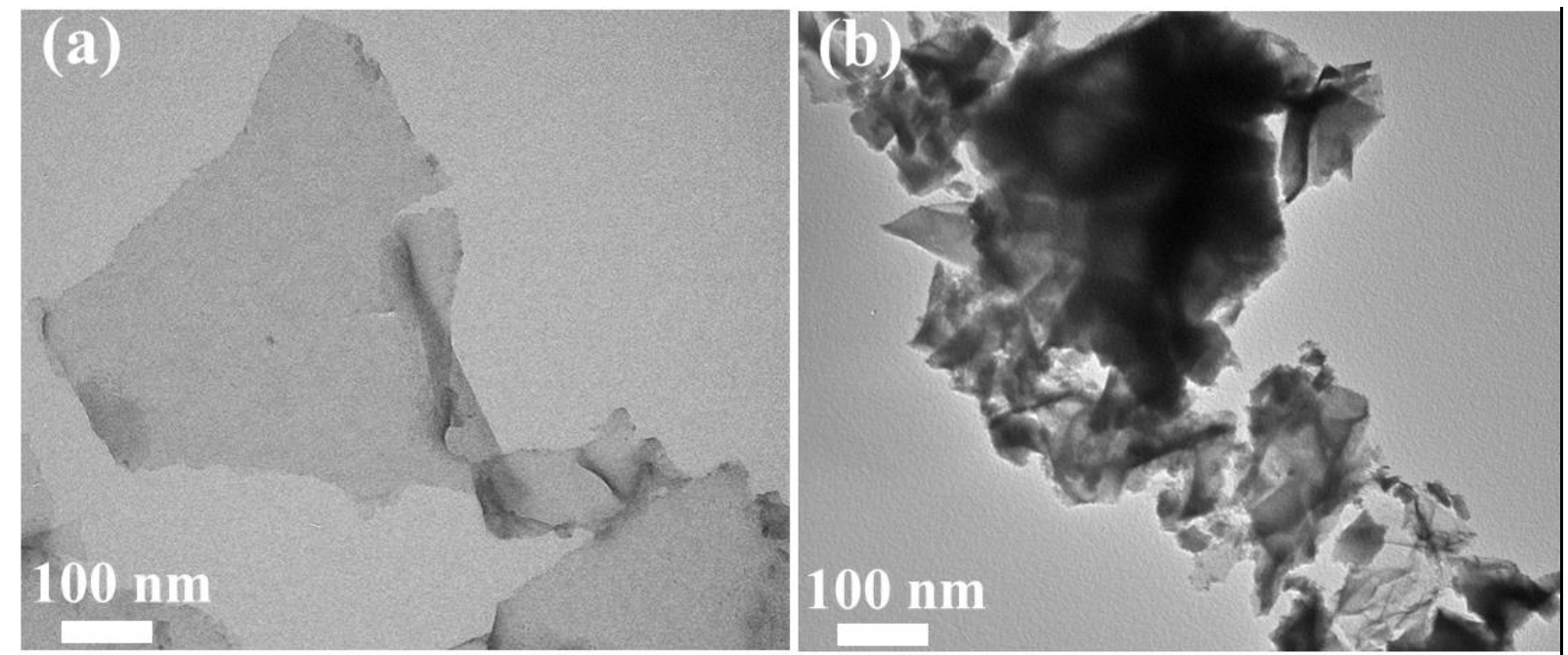

(c)

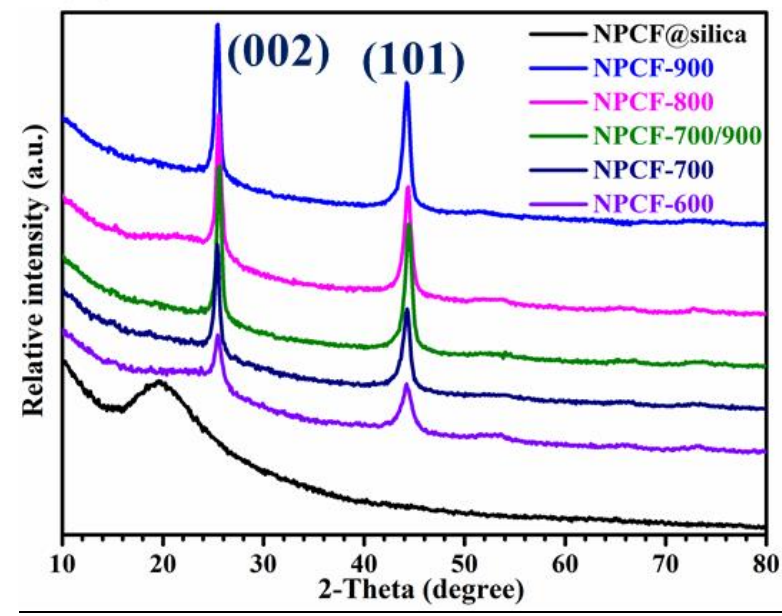

(d)

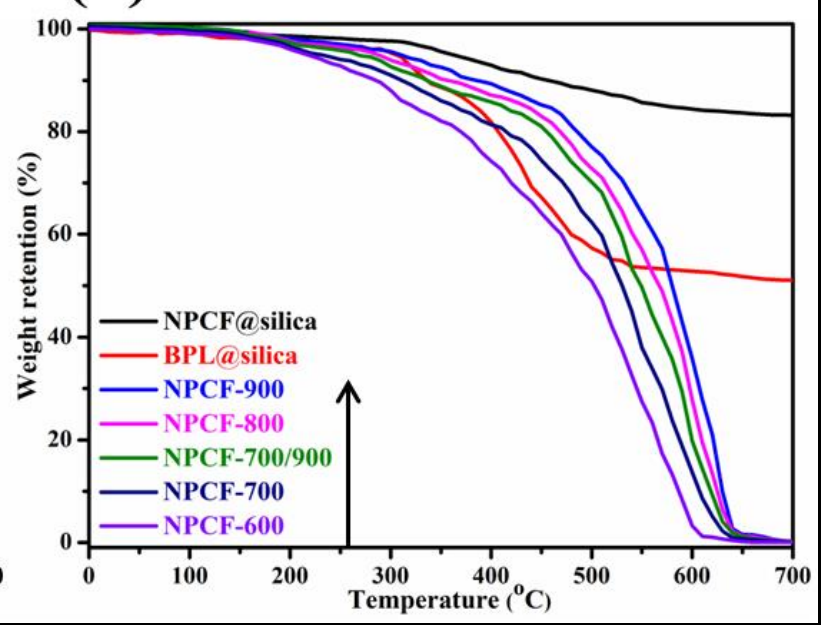

Figure S3. TEM images of (a) BPL flake and (b) BPL@silica hybrid. (c) XRD patterns and (d) TGA curves of NPCFs before and after the removal of the silica template. 


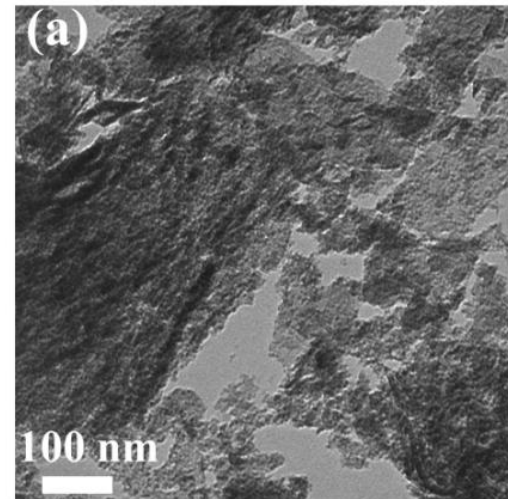

(c)

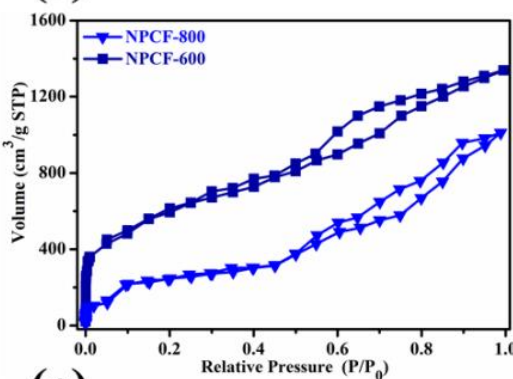

(e)

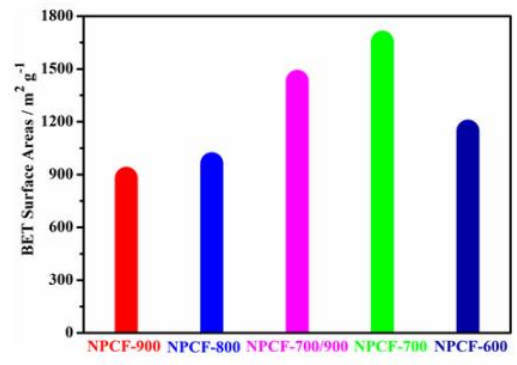

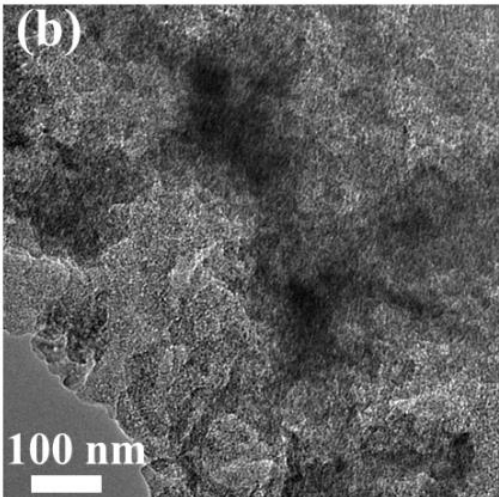

(d)

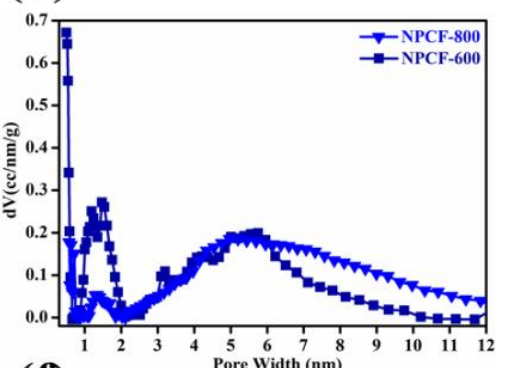

(f)

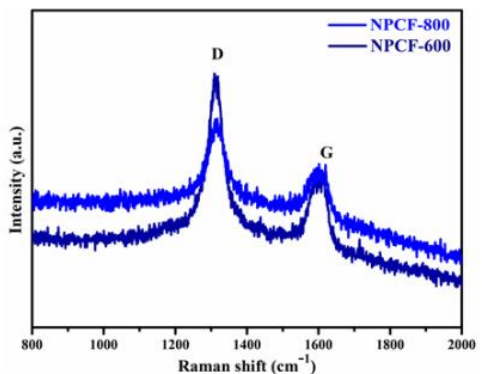

Figure S4. TEM images of (a) NPCF-600 and (b) NPCF-800. The corresponding (c) $\mathrm{N}_{2}$ adsorption/desorption isotherms and (d) pore size spread plots. (e) Histogram of BET surface areas of NPCFs. (f) Raman curves of NPCF-600 and NPCF-800.

(a)

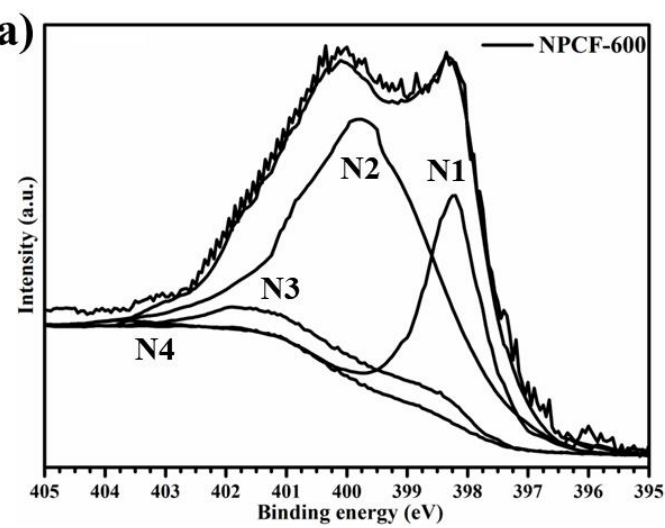

(b)

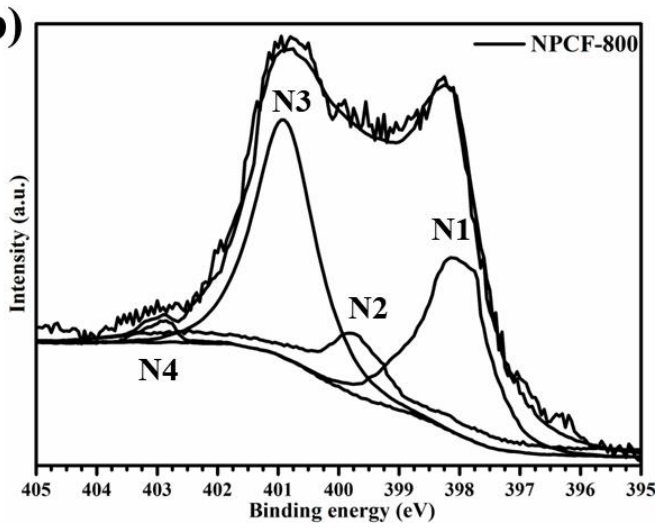

Figure S5. High resolution N1s XPS spectra of (a) NPCF-600 and (b) NPCF-800. 
(a)

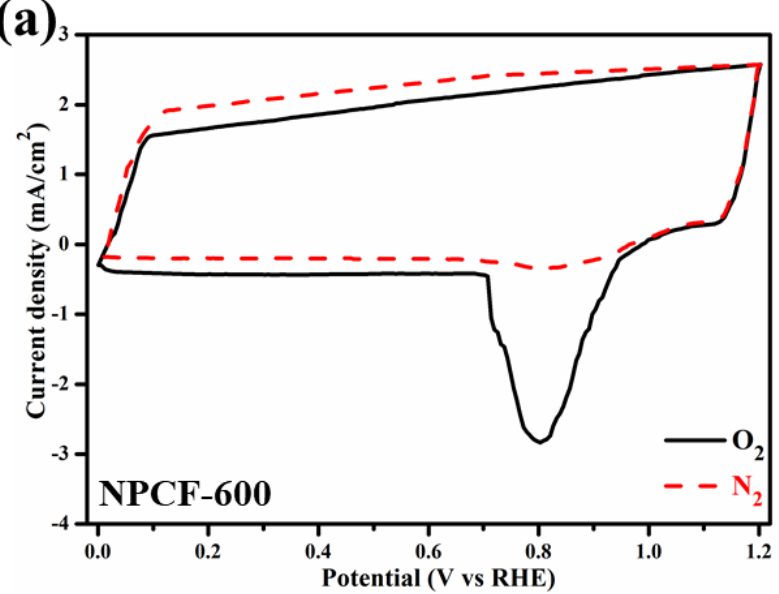

(b)

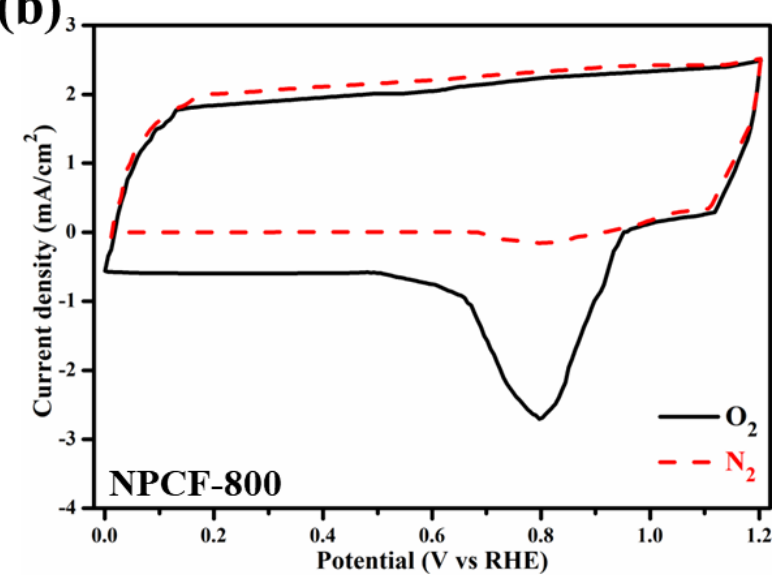

Figure S6. CV curves of (a) NPCF-600 and (b) NPCF-800 electrodes in $\mathrm{O}_{2}$ or $\mathrm{N}_{2}$ saturated $0.1 \mathrm{M} \mathrm{KOH}$.

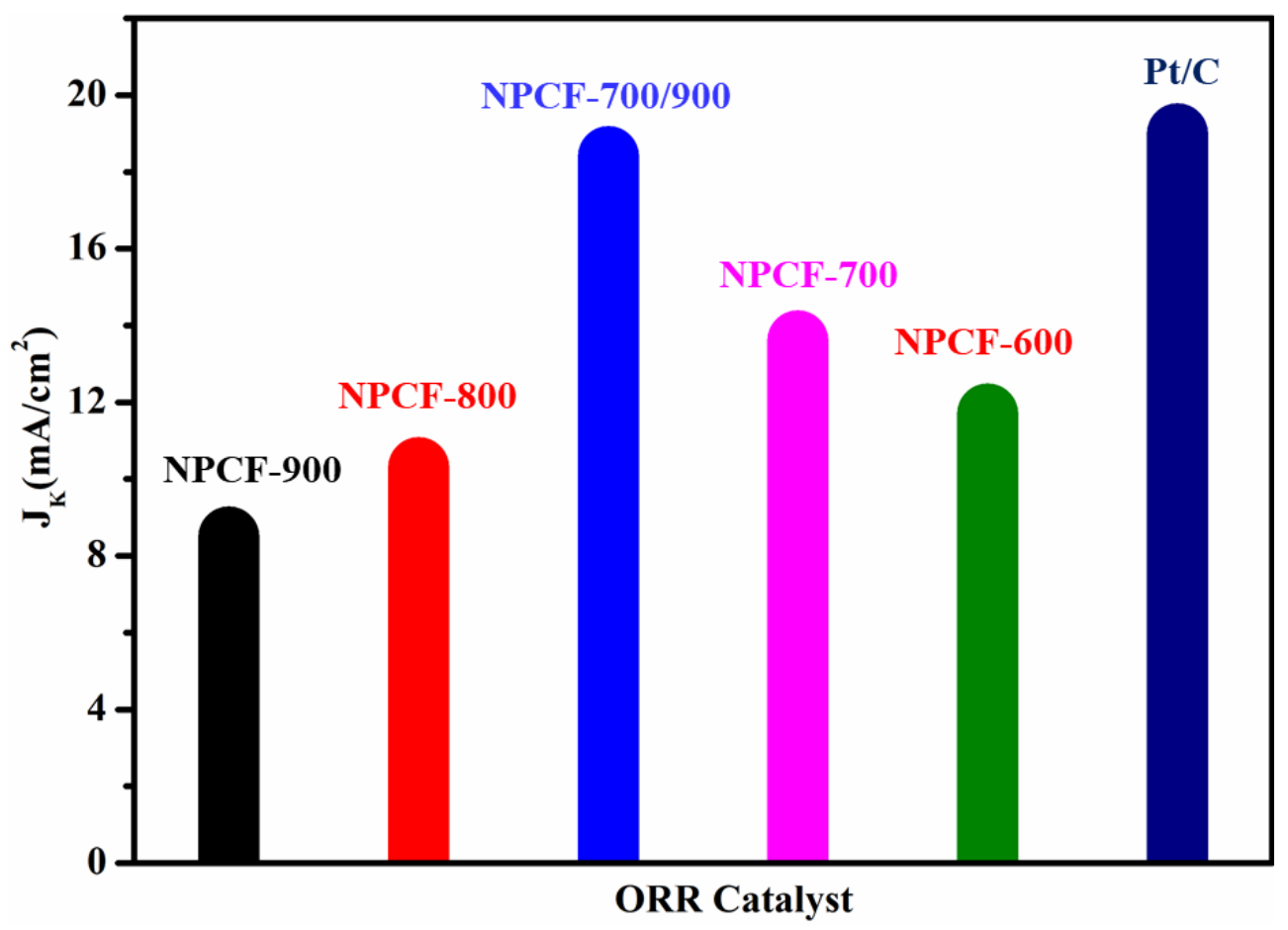

Figure S7. The kinetic-limiting current density $\left(J_{\mathrm{K}}\right)$ of different samples. 


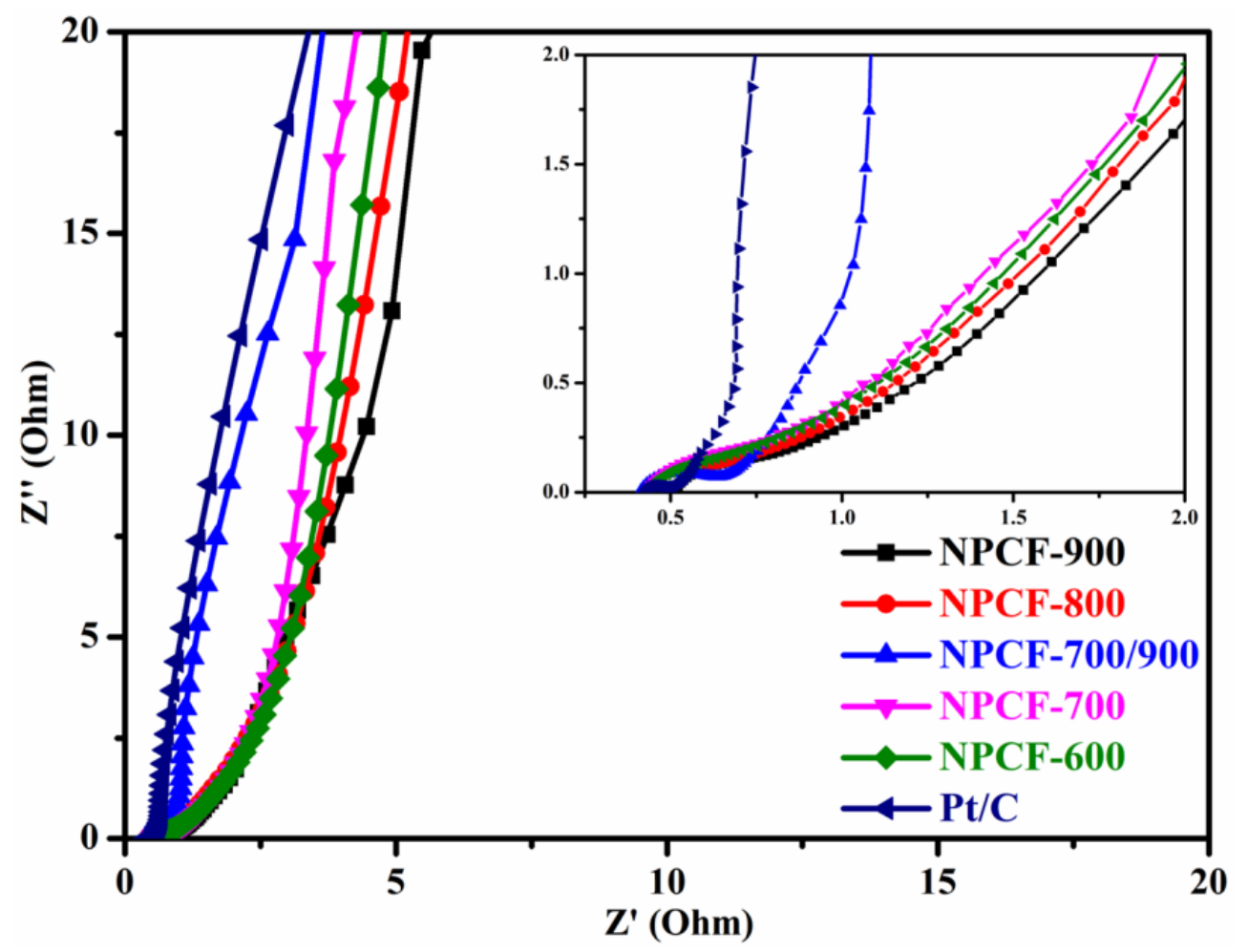

Figure S8. Nyquist plots of NPCFs with inset of magnified details.

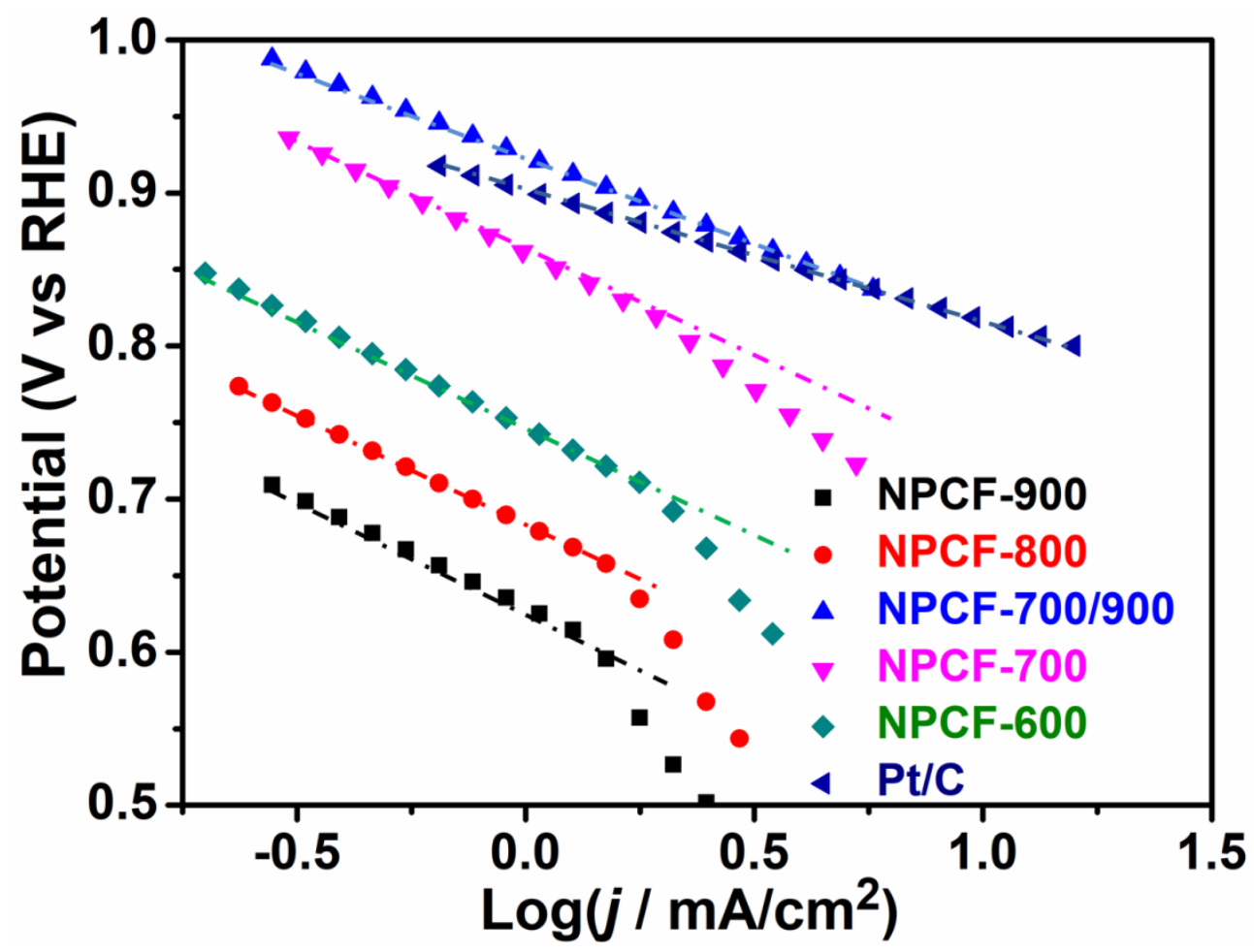

Figure S9. Tafel slope plots derived from the corresponding LSV curves at $1600 \mathrm{rpm}$ in $\mathrm{O}_{2^{-}}$ saturated $0.1 \mathrm{M} \mathrm{KOH}$ electrolyte of various catalysts of the samples and $\mathrm{Pt} / \mathrm{C}$. 


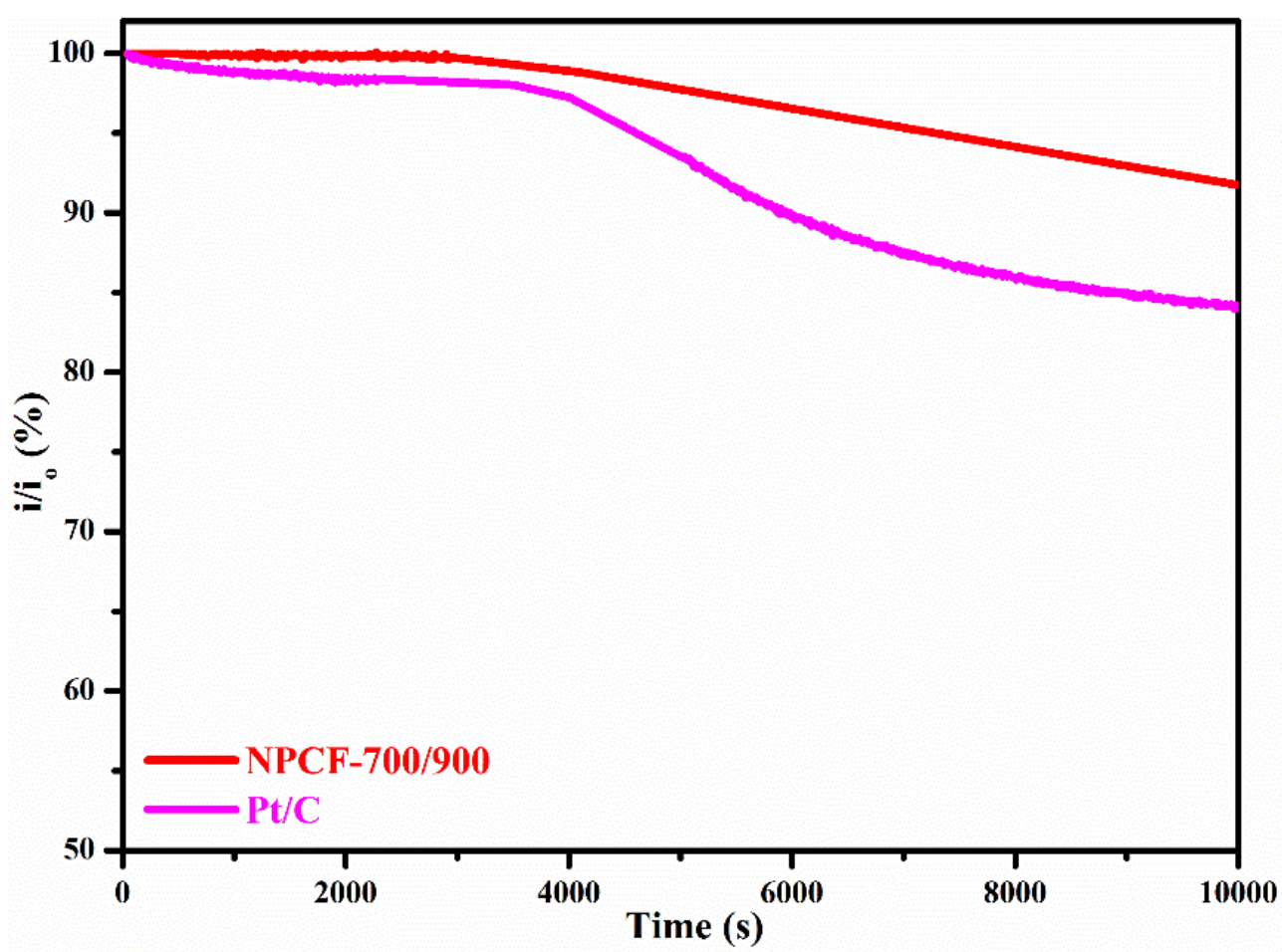

Figure S10. The i-t chronoamperometric response of NPCF-700/900 and $\mathrm{Pt} / \mathrm{C}$ after exposure to oxygen at $0.7 \mathrm{~V}$ for $10000 \mathrm{~s}$.

Table S1. Summary of the properties and ORR performance for NPCF-700/900 and some metal-free catalysts reported previously. All the catalysts were tested in $0.1 \mathrm{M} \mathrm{KOH}$ solution.

\begin{tabular}{|c|c|c|c|c|c|c|c|c|c|}
\hline Materials & Heteroatom & $\begin{array}{c}S_{\text {BET }} \\
\left(\mathrm{m}^{2} \mathrm{~g}^{-1}\right)\end{array}$ & $\begin{array}{l}\text { Reduction } \\
\text { peak }(V)\end{array}$ & $\begin{array}{c}E_{\text {onset }} \\
\text { (V) }\end{array}$ & $\begin{array}{l}E_{1 / 2} \\
(V)\end{array}$ & $\begin{array}{c}J_{L}(\mathbf{m A} \\
\left.\mathrm{cm}^{-2}\right)\end{array}$ & $\begin{array}{c}J_{K}(\mathbf{m A} \\
\left.\mathbf{c m}^{-2}\right)\end{array}$ & $n$ & Refs \\
\hline NPCF-700/900 & $8.3 \mathrm{~N}$ at $\%$ & 1431 & 0.809 & 0.93 & 0.83 & 4.89 & 18.4 & 3.95 & Present work \\
\hline $\mathrm{Pt} / \mathrm{C}$ & -- & -- & 0.803 & 0.91 & 0.81 & 4.95 & 19 & 3.97 & Present work \\
\hline $\begin{array}{c}\mathbf{N}_{0.54}-Z_{3} / M_{1^{-}} \\
900\end{array}$ & $3.62 \mathrm{~N}$ at $\%$ & 1394 & 0.88 & 0.94 & 0.824 & 4.3 & -- & 3.97 & $\begin{array}{c}\text { Energy Environ. Sci. } \\
2019,12,648\end{array}$ \\
\hline NCN-1000-5 & $2.46 \mathrm{~N}$ at $\%$ & 1793 & 0.86 & 0.95 & 0.82 & 6.43 & 50 & 3.92 & $\begin{array}{c}\text { Energy Environ. Sci. } \\
2019,12,322\end{array}$ \\
\hline NOPHC $_{10-900}$ & $\begin{array}{c}6.63 \mathrm{~N} \text { at } \%, 1.11 \\
\mathrm{P} \text { at } \%\end{array}$ & $\sim 250$ & 0.67 & 0.9 & 0.77 & 4.2 & -- & 3.8 & $\begin{array}{c}\text { Appl. Catal. B - } \\
\text { Environ. 2019, 248, } \\
239\end{array}$ \\
\hline N-HsG DY-900 & $2.9 \mathrm{~N}$ at $\%$ & 1754 & 0.85 & 1.02 & 0.85 & 6.5 & -- & 3.92 & $\begin{array}{c}\text { Nat. Commun. 2018, } \\
\qquad, 3376\end{array}$ \\
\hline B ClCNTs-2 & $1.17 \mathrm{~B}$ at $\%, 1.8$ & -- & 0.811 & 0.95 & 0.84 & 5.5 & -- & 3.6 & ACS Energy Lett. \\
\hline
\end{tabular}




\begin{tabular}{|c|c|c|c|c|c|c|c|c|c|}
\hline PNGF(op) & $\begin{array}{c}4 \mathrm{~N} \text { at } \%, 2.32 \mathrm{P} \\
\text { at } \%\end{array}$ & -- & -- & $\sim 0.95$ & 0.845 & $\sim 5.5$ & & 3.91 & $\begin{array}{c}\text { Energy Environ. Sci. } \\
2017,10,1186\end{array}$ \\
\hline PNPC-1000 & $7.6 \mathrm{~N}$ at $\%$ & $\sim 1180$ & 0.72 & 0.89 & 0.76 & 6.9 & -- & 3.82 & $\begin{array}{c}\text { J. Mater. Chem. A, } \\
2017,5,789\end{array}$ \\
\hline SHG & $3.8 \mathrm{~N}$ at\% & 576 & 0.88 & 1.01 & 0.87 & 5.1 & -- & 3.96 & $\begin{array}{c}\text { Adv. Mater. 2017, } \\
29,1604942\end{array}$ \\
\hline $\begin{array}{l}\text { NPC }-1000- \\
2.08\end{array}$ & $2.08 \mathrm{~N}$ at $\%$ & 811 & 0.828 & 1.02 & 0.902 & 5.85 & & $\sim 4$ & $\begin{array}{l}\text { Adv. Funct. Mater. } \\
2017,27,1606190\end{array}$ \\
\hline $\begin{array}{l}\text { NHPC-900- } \\
1000\end{array}$ & $4.4 \mathrm{~N}$ at $\%$ & 2412 & 0.88 & 0.96 & 0.84 & $\sim 5.2$ & $\sim 4$ & 4 & $\begin{array}{c}\text { ACS Catal. 2017, 7, } \\
6082\end{array}$ \\
\hline N,S-CN & $\begin{array}{c}4.1 \mathrm{~N} \text { at } \%, 6.1 \mathrm{~S} \\
\text { at } \%\end{array}$ & 273 & -0.22 & -0.04 & -0.2 & $\sim 4.3$ & 16 & 3.98 & $\begin{array}{c}\text { Nano Energy 2016, } \\
\qquad 19,373\end{array}$ \\
\hline NSC & $\begin{array}{c}4.5 \mathrm{~N} \text { at } \%, 0.2 \mathrm{~S} \\
\text { at } \%\end{array}$ & 1608 & 0.75 & 0.87 & 0.74 & 5.1 & -- & 4 & $\begin{array}{l}\text { Adv. Funct. Mater. } \\
2016,26,8651\end{array}$ \\
\hline NC-3 & $7.3 \mathrm{~N}$ at $\%$ & 1666 & 0.72 & 0.84 & 0.71 & 4.8 & -- & 3.8 & $\begin{array}{l}\text { Adv. Funct. Mater. } \\
\text { 2016, 26, } 8651\end{array}$ \\
\hline N/S-2DPC-60 & $\begin{array}{c}5.39 \mathrm{~N} \text { wt } \%, 0.26 \\
\mathrm{~S} \text { wt } \%\end{array}$ & 953 & 0.65 & 0.86 & 0.75 & $\sim 5$ & 19.2 & 3.9 & $\begin{array}{c}\text { Adv. Funct. Mater. } \\
2016,26,5893\end{array}$ \\
\hline $\begin{array}{l}\text { MPSA/GO- } \\
1000\end{array}$ & -- & -- & -- & 0.92 & 0.82 & $\sim 5.3$ & 16.9 & 3.7 & $\begin{array}{l}\text { Angew. Chem. Int. } \\
\text { Ed. 2016, 55, } 2230\end{array}$ \\
\hline N,P-CGHNs & $3.3 \mathrm{~N}$ at $\%$ & 194 & 0.82 & 0.94 & 0.82 & 5.6 & -- & 3.9 & $\begin{array}{l}\text { Adv. Mater. 2016, } \\
\qquad 28,4606\end{array}$ \\
\hline N-C/CNT & $2.75 \mathrm{~N}$ at $\%$ & 249 & -- & 0.97 & 0.83 & $\sim 5.5$ & -- & 3.8 & $\begin{array}{c}\text { ACS Catal. 2016, 6, } \\
1408\end{array}$ \\
\hline NPMC-1000 & $1.8 \mathrm{~N}$ at $\%$ & 1663 & 0.86 & 0.94 & 0.85 & 4.3 & $\sim 26$ & 3.94 & $\begin{array}{c}\text { Nature Nanotech. } \\
2015,10,444\end{array}$ \\
\hline $\begin{array}{c}\text { macro/meso- } \\
\qquad \mathrm{NC}-\mathrm{NH}_{3}\end{array}$ & $6 \mathrm{~N}$ wt $\%$ & 1462 & -- & -0.1 & -0.2 & 6.6 & -- & 3.99 & $\begin{array}{c}\text { Energy Environ. Sci. } \\
2015,8,3274\end{array}$ \\
\hline N,P-G CNS & $\begin{array}{c}4.71 \mathrm{~N} \text { at } \%, 1.72 \\
\mathrm{P} \text { at } \%\end{array}$ & 900.2 & 0.85 & 1.01 & 0.86 & 5.56 & -- & 3.96 & $\begin{array}{c}\text { ACS Catal. 2015, 5, } \\
4133\end{array}$ \\
\hline CNC700 & -- & 1713 & $\sim 0.02$ & 0.11 & $\sim 0$ & $\sim 4.3$ & -- & 2.9 & $\begin{array}{c}\text { ACS Catal. 2015, 5, } \\
6707\end{array}$ \\
\hline NGSH & $0.58 \mathrm{~N}$ at $\%$ & 812.9 & $\sim 0.64$ & 0.88 & 0.68 & $\sim 4.8$ & 132.8 & 3.22 & Small 2014, 10,2251 \\
\hline NG-1000 & $3.2 \mathrm{~N}$ at $\%$ & 185 & -0.27 & -0.06 & -0.25 & 3.8 & 11.9 & 3.89 & $\begin{array}{c}\text { Nano Energy 2014, } \\
\qquad 3,55\end{array}$ \\
\hline
\end{tabular}




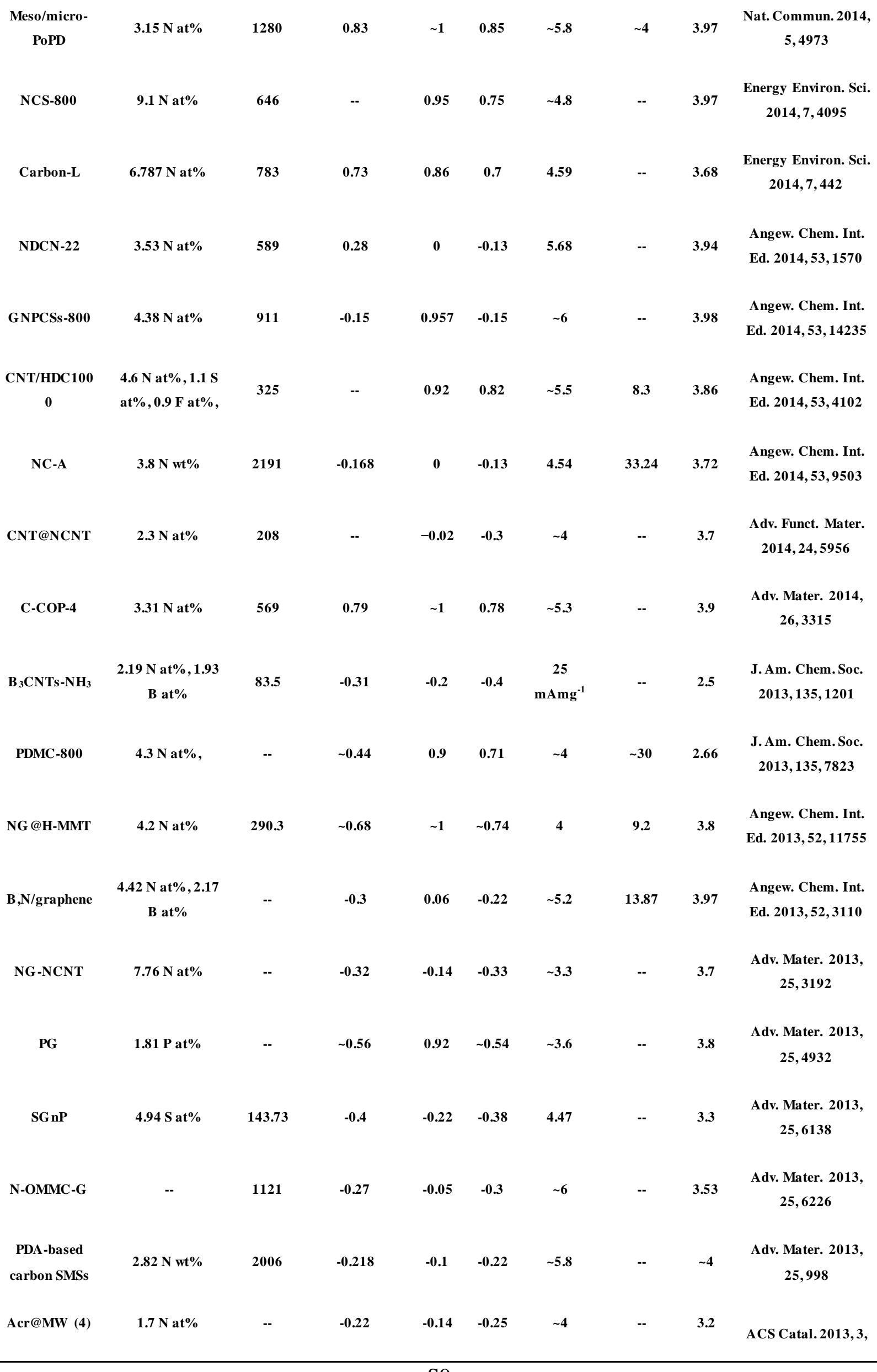




\begin{tabular}{|c|c|c|c|c|c|c|c|c|c|}
\hline NT-G & $\begin{array}{c}5.4 \mathrm{~N} \text { at } \%, 0.03 \\
\text { Fe at } \%\end{array}$ & -- & 0.75 & $\sim 0.89$ & $\sim 0.76$ & $\sim 5.5$ & $\sim 20$ & $\sim 4$ & $\begin{array}{l}\text { Nat. Nanotechnol. } \\
\quad 2012,7,394\end{array}$ \\
\hline POMC-3 & $1.36 \mathrm{P}$ at $\%$ & 1182 & -0.19 & -0.11 & -0.2 & $\sim 5.3$ & -- & 3.91 & $\begin{array}{l}\text { J. Am. Chem. Soc. } \\
2012,134,16127\end{array}$ \\
\hline CA-TCA-900 & $\begin{array}{c}4.96 \mathrm{~N} \text { at } \%, 0.74 \\
\mathrm{~S} \text { at } \%\end{array}$ & 224.5 & -0.35 & -0.13 & -0.28 & $\sim 1.8$ & -- & $\sim 4$ & $\begin{array}{c}\text { Green Chem. 2012, } \\
\qquad 14,1515\end{array}$ \\
\hline N-RG-O 1000 & $5.2 \mathrm{~N}$ at $\%$ & $\sim 350$ & -- & -0.1 & -0.3 & $\sim 7.5$ & -- & 2.85 & $\begin{array}{c}\text { Energy Environ. Sci. } \\
2012,5,7936\end{array}$ \\
\hline GF & $4.9 \mathrm{~N}$ at $\%$ & $\sim 280$ & -0.3 & -0.18 & -0.38 & $\sim 1.5$ & -- & 3.7 & $\begin{array}{l}\text { Angew. Chem. Int. } \\
\text { Ed. 2012, 51, } 11371\end{array}$ \\
\hline N-S-G & $\begin{array}{c}4.5 \mathrm{~N} \text { at } \%, 2 \mathrm{~S} \\
\text { at } \%\end{array}$ & $\sim 220$ & -0.24 & -0.06 & -0.33 & $\sim 10$ & 15 & 3.6 & $\begin{array}{l}\text { Angew. Chem. Int. } \\
\text { Ed. 2012, 51, } 11496\end{array}$ \\
\hline $150-\mathrm{C} / \mathrm{CN}$ & $21.03 \mathrm{~N}$ wt $\%$ & 97 & -0.3 & -0.14 & -0.32 & $\sim 4.8$ & -- & 2.89 & $\begin{array}{l}\text { Angew. Chem. Int. } \\
\text { Ed. 2012, 51, 3892 }\end{array}$ \\
\hline $\mathbf{B}_{12} \mathbf{C}_{77} \mathbf{N}_{11}$ & $\begin{array}{c}12 \mathrm{~N} \text { at } \%, 11 \mathrm{~B} \\
\text { at } \%\end{array}$ & -- & -0.28 & $\mathbf{0}$ & -0.25 & $\sim 5.5$ & -- & 4 & $\begin{array}{l}\text { Angew. Chem. Int. } \\
\text { Ed. 2012, 51, } 4209\end{array}$ \\
\hline NCNC700/900 & $7.1 \mathrm{~N}$ at $\%$ & 884 & -0.22 & -0.13 & -0.28 & $\begin{array}{c}\sim 41 \\
\mathrm{mAmg}^{-1}\end{array}$ & -- & 3.27 & $\begin{array}{l}\text { Adv. Mater. 2012, } \\
\qquad 24,5593\end{array}$ \\
\hline SG900 & $1.2 \mathrm{~S}$ at $\%$ & -- & -- & -0.18 & -0.4 & -- & 5.3 & 3.2 & $\begin{array}{l}\text { Adv. Funct. Mater. } \\
\text { 2012, 22, } 3634\end{array}$ \\
\hline NG900 & $4.6 \mathrm{~N}$ at $\%$ & 816 & -0.32 & $\mathbf{0}$ & -0.3 & -- & 7.8 & $\sim 4$ & $\begin{array}{l}\text { Adv. Funct. Mater. } \\
\text { 2012, 22, } 3634\end{array}$ \\
\hline NCNT-1000 & $6 \mathrm{~N}$ at $\%$ & -- & -0.26 & -0.1 & -0.25 & 2.8 & -- & $\sim 2.9$ & $\begin{array}{c}\text { ACS Nano 2012, 6, } \\
8904\end{array}$ \\
\hline NG -900 & $5 \mathrm{~N}$ at $\%$ & 508 & -0.16 & $\mathbf{0}$ & -0.17 & $\sim 4$ & 6.67 & 3.7 & $\begin{array}{c}\text { ACS Nano 2012, 6, } \\
9541\end{array}$ \\
\hline $\begin{array}{l}\text { S-graphene- } \\
1050\end{array}$ & $1.3 \mathrm{~S} \mathrm{wt} \%$ & 435 & -0.3 & -0.06 & -0.21 & $\sim 7.2$ & 9.34 & 3.82 & $\begin{array}{c}\text { ACS Nano 2012, 6, } \\
205\end{array}$ \\
\hline PDDA-ACNT & $3.8 \mathrm{~N}$ at $\%$ & & -0.28 & -0.09 & -0.3 & $\sim 4.5$ & -- & 3.72 & $\begin{array}{c}\text { J. Am. Chem. Soc. } \\
2011,133,5182\end{array}$ \\
\hline $\begin{array}{c}\text { g- } \\
\mathrm{C}_{3} \mathrm{~N}_{4} @ \mathbf{C M K}-3\end{array}$ & -- & 623 & -0.25 & -0.1 & -0.18 & $\sim 3.5$ & 11.3 & 4 & $\begin{array}{l}\text { J. Am. Chem. Soc. } \\
\text { 2011, 133, } 20116\end{array}$ \\
\hline meso-EmG & $12.2 \mathrm{~N}$ wt $\%$ & 1553 & -0.192 & $\sim 0.05$ & -0.1 & $\sim 6$ & -- & 3.2 & $\begin{array}{l}\text { J. Am. Chem. Soc. } \\
\text { 2011, 133, } 206\end{array}$ \\
\hline VA-BCN & $\begin{array}{c}10.3 \mathrm{~N} \text { at } \%, 4.2 \mathrm{~B} \\
\text { at } \%\end{array}$ & -- & -0.32 & -0.1 & -0.25 & $\sim 4.5$ & 10.13 & $\sim 4$ & $\begin{array}{l}\text { Angew. Chem. Int. } \\
\text { Ed. 2011, 50,11756 }\end{array}$ \\
\hline
\end{tabular}




\begin{tabular}{|c|c|c|c|c|c|c|c|c|c|}
\hline $\begin{array}{c}\text { P-doped } \\
\text { graphite/GC }\end{array}$ & $0.26 \mathrm{P}$ at $\%$ & -- & -0.3 & 0.1 & -0.35 & $\sim 5.6$ & -- & $\sim 3$ & $\begin{array}{l}\text { Angew. Chem. Int. } \\
\text { Ed. 2011, 50,3257 }\end{array}$ \\
\hline G-CN800 & $10 \mathrm{~N}$ at $\%$ & 542 & -0.25 & -0.1 & -0.25 & $\sim 3.8$ & 7.3 & 4 & $\begin{array}{l}\text { Angew. Chem. Int. } \\
\text { Ed. 2011, 50, 5339 }\end{array}$ \\
\hline $\mathrm{B}_{3} \mathrm{CNTs}$ & 2.24 B at $\%$ & -- & -0.35 & -0.28 & -0.4 & $\begin{array}{c}\sim 17 \\
\mathrm{mAmg}^{-1}\end{array}$ & -- & 2.5 & $\begin{array}{l}\text { Angew. Chem. Int. } \\
\text { Ed. 2011, 50, } 7132\end{array}$ \\
\hline $\begin{array}{l}\text { PDDA- } \\
\text { G raphene }\end{array}$ & -- & -- & -0.35 & -0.12 & -0.4 & $\sim 2.8$ & $\sim 8$ & $\sim 4$ & $\begin{array}{c}\text { ACS Nano 2011, } 5 \text {, } \\
6202\end{array}$ \\
\hline NCNTs & $3.6 \mathrm{~N}$ at $\%$ & -- & 0.2 & $\sim 0.5$ & $\sim 0.2$ & $\sim 2.1$ & 3.54 & 3.92 & $\begin{array}{l}\text { J. Am. Chem. Soc. } \\
\text { 2010, 132, } 15127\end{array}$ \\
\hline N-OMC-1050 & $4.6 \mathrm{~N}$ at $\%$ & 1923 & -- & 0.72 & 0.62 & $\sim 4.2$ & -- & 3.41 & $\begin{array}{c}\text { Chem. Mater. 2010, } \\
\text { 22,2178 }\end{array}$ \\
\hline PDI-900/GC & $2.7 \mathrm{~N}$ at $\%$ & 510 & -0.26 & -0.13 & -0.26 & $\sim 6.1$ & 9.15 & 3.89 & $\begin{array}{l}\text { Angew. Chem. Int. } \\
\text { Ed. 2010, 49, } 2565\end{array}$ \\
\hline N-graphene & $4 \mathrm{~N}$ at $\%$ & -- & -- & -0.18 & -0.3 & 0.8 & -- & 4 & $\begin{array}{c}\text { ACS Nano 2010,4, } \\
1321\end{array}$ \\
\hline
\end{tabular}

